\title{
WEAK SOLUTIONS OF THE POROUS MEDIUM EQUATION
}

\author{
BJÖRN E. J. DAHLBERG AND CARLOS E. KENIG
}

\begin{abstract}
We show that if $u \geq 0, u \in L_{\text {loc }}^{m}(\Omega), \Omega \subset \mathbf{R}^{n+1}$ solves $\partial u / \partial t=$ $\Delta u^{m}, m>1$, in the sense of distributions, then $u$ is locally Hölder continuous in $\Omega$.
\end{abstract}

\section{INTRODUCTION}

The purpose of this article is to study the regularity properties for nonnegative solutions of the porous medium equation.

The porous medium equation

$$
\frac{\partial u}{\partial t}=\Delta u^{m}, \quad m>1
$$

models the gas flow in a porous medium and has recently been the subject of extensive studies by a large number of researchers. We want here to single out the results by Aronson and Caffarelli [1], Benilan, Crandall and Pierre [2], Dahlberg and Kenig [4, 5] on the initial value problem for the equation (1). By combining the results of these papers, one has the very unusual situation that the understanding of the initial value problem for the nonlinear equation (1) is on the same level as for the corresponding problem for the linear case, i.e. the heat equation $\partial u / \partial t=\Delta u$.

In particular, a necessary and sufficient growth condition has been established on a nonnegative measure $\mu$ on $\mathbf{R}^{n}$ in order for the initial value problem to be solvable on $\mathbf{R}^{n} \times(0, \infty)$ with initial data $\mu$. This growth condition is that

$$
\mu(|x|<R)=o\left(R^{n+2 /(m-1)}\right) \quad \text { as } R \rightarrow \infty .
$$

Furthermore, the initial value problem has a unique solution in the class of all nonnegative, continuous weak solutions.

We want to remark that for the heat equation the corresponding growth condition is that

$$
\int_{\mathbf{R}^{n}} e^{-\sigma|x|^{2}} d \mu(x)<\infty
$$

for all $\sigma>0$.

In view of the above results it is natural to ask if the uniqueness continues to hold under weaker conditions on the solution. This question is of course

Received by the editors November 2, 1988 and, in revised form, December 21, 1990.

1980 Mathematics Subject Classification (1985 Revision). Primary 35K55.

The work of the second author was supported in part by the NSF and the J. S. Guggenheim Foundation. 
a special case of the more general problem of understanding whether a weak solution is continuous.

We will say that $u$ is a weak solution of the porous medium equation in the domain $\Omega$ if $u \in L_{\mathrm{loc}}^{m}(\Omega), u \geq 0$, and $u$ solves equation (1) in the distribution sense.

We can now state our main result.

Theorem 1.1. Suppose $u \geq 0$ is a weak solution of the porous medium equation (1) in the domain $\Omega$. Then there is a continuous weak solution $u^{*}$ of $(1)$ in $\Omega$ such that $u=u^{*}$ a.e. in $\Omega$.

We want to point out that simple examples show that $u$ need not be smoother than Hölder continuous. It is furthermore known, see DiBenedetto and Friedman [6], that each continuous solution is Hölder continuous.

The proof of the theorem follows the following strategy:

We first show that for all cylinders $\omega=D \times(a, b), \omega \subset \subset \Omega$, with $D$ a bounded smooth domain, the solution $u$ has a trace on $\partial \omega$. Then we construct a continuous weak solution $u^{*}$ in $\omega$ whose trace on the parabolic boundary $\partial_{p} \omega=\partial \omega \backslash D \times\{b\}$ equals the trace of $u$.

In order to show that $u=u^{*}$ a.e. in $\omega$ the next step consists in showing that there is a function $S=S(x)$ in $\omega$ such that if we set

$$
w(x, t)=S(x)-\int_{a}^{t} u^{m}(x, s) d s, \quad w^{*}(x, t)=S(x)-\int_{a}^{t}\left(u^{*}\right)^{m}(x, s) d s,
$$

then $w$ and $w^{*}$ solve the equation $\partial W / \partial t=\varphi(\Delta W)$. Here $\varphi(u)=\operatorname{sign}(u)|u|^{m}$. Using elliptic regularity theory we conclude that $w$ and $w^{*}$ are bounded. From the boundedness we then deduce the equality between $w$ and $w^{*}$ by observing that $w-w^{*}$ satisfies a linear parabolic equation with variable coefficients with data zero on $\partial_{p} \omega$. An important part of the argument here is to analyze the energy norm of the difference $w-w^{*}$. Since $\Delta w=-u$ and $\Delta w^{*}=-u^{*}$ the result then follows.

\section{INHOMOGENEOUS BOUNDARY CONDITIONS}

We will now assume $D \subset \mathbf{R}^{n}$ is bounded with smooth boundary. Let $d \sigma$ denote the surface measure of $\partial D$ and let $\delta(x)$ denote the distance from $x$ to the boundary. Let $0<T<\infty$ and set $\Omega=D \times I$ where $I=(0, T)$. Let $m>1$. We are interested in the problem

$$
\left\{\begin{array}{l}
u \geq 0, u \in L_{\mathrm{loc}}^{m}(\Omega), \\
\frac{\partial u}{\partial t}=\Delta u^{m} \text { in the distribution sense in } \Omega, \\
u(x, 0)=f(x), \\
(u(P))^{m}=g(P), \quad P \in \partial D \times(0, T) .
\end{array}\right.
$$

We have the following a priori inequality.

Proposition 2.1. Assume $u \in C(\bar{\Omega})$ and solves (2). Suppose

$$
\int_{D} \delta f d x \leq L \text { and } \iint_{S} g d \sigma d t \leq L
$$

where $S=\partial D \times I$. Then

$$
\iint_{\Omega} u^{m} d x d t \leq C(L)
$$




$$
\sup _{t \in I} \int_{D} \delta(x) u(x, t) d x \leq C(L) .
$$

Proof. Let $\eta$ be defined by $\Delta \eta=-1$ in $D$ and $\eta=0$ on $\partial D$. Then $c_{1} \delta \leq \eta \leq$ $c_{2} \delta$. Let $\partial / \partial n$ denote differentiation with respect to the inward unit normal. By the Green formula

$$
\begin{aligned}
& \frac{\partial}{\partial t} \int_{D} u(x, t) \eta(x) d x=\int_{D} \eta \Delta u^{m} d x \\
& \quad=-\int_{D}(u(x, t))^{m} d x+\int_{\partial D}(u(x, t))^{m} \frac{\partial \eta}{\partial n} d \sigma(x) .
\end{aligned}
$$

Hence for $t \in I$

$$
\int_{D} \eta(x) u(x, t) d x \leq \int_{D} \eta(x) u(x, 0) d x+\iint_{S} \frac{\partial \eta(x)}{\partial n} g(x, t) d \sigma(x)
$$

which takes care of (4). Since we now have (4) it is easily seen that (3) follows by integrating (5) in $t$ from 0 to $T$.

Now we want to study the initial value problem (2) with data being measures. Let $\mathscr{G}$ be the class of pairs $(\mu, \lambda)$ of nonnegative measures on $D$ and $S=$ $\partial D \times(0, T)$ respectively such that

$$
\int_{D} \delta d \mu+\iint_{S} d \lambda<\infty
$$

Let $\mathscr{M}=\mathscr{M}(D, T)$ be the class of $\eta \in C^{\infty}\left(\mathbf{R}^{n+1}\right)$ with the property that $\eta(x, t)=0$ for $x \in \partial D$ or $t \geq \tau$ for some $\tau \in(0, T)$. Suppose $(\mu, \lambda) \in \mathscr{G}$. We say that $u \geq 0$ is a continuous weak solution of $(2)$ with data $(\mu, \lambda)$ if

1. $u$ is continuous in $\Omega$,

2. $\iint_{\Omega} u^{m} d x d t<\infty$,

3. for all $\eta \in \mathscr{M}$ we have that

$$
\iint_{\Omega}\left(u^{m} \Delta \eta+u \frac{\partial \eta}{\partial t}\right) d x d t+\int_{D} \eta(x, 0) d \mu+\iint_{S} \frac{\partial \eta}{\partial n} d \lambda=0
$$

\section{WEAK SOLUTIONS}

In this section we will assume that $u \geq 0$ and $u \in L_{\text {loc }}^{m}(\Omega)$ where $\Omega$ is a domain in $\mathbf{R}^{n+1}$. We will also assume that $u$ solves the equation $\partial u / \partial t=\Delta u^{m}$ in the distribution sense. The conjecture is that $u$ is continuous. Our starting point is the following couple of observations. We begin by establishing the existence of trace at each time level.

Lemma 3.1. Let $I=(a, b)$ be an interval and let $D \subset \mathbf{R}^{n}$ be a bounded domain with smooth boundary. Set $K=\bar{D}$ and assume that $K \times[a, b]$ is compact and contained in $\Omega$. Then for each $\tau \in I$ there is a unique bounded and nonnegative measure $\nu_{\tau}$ on $D$ such that

$$
\sup _{\tau \in I} \int_{D} d \nu_{\tau}<\infty
$$

and

$$
\iint_{\Omega \cap\{t>\tau\}}\left(u^{m} \Delta \eta+u \frac{\partial \eta}{\partial t}\right) d x d t+\int_{D} \eta(x, \tau) d \nu_{\tau}=0
$$

for each $\eta \in C_{0}^{\infty}(\omega)=\mathscr{A}$. Here $\omega=D \times(a, b)$. 
Proof. For $\tau \in I$ and $\eta \in \mathscr{A}$ set

$$
\Lambda_{\tau}(\eta)=\iint_{\Omega \cap\{t>\tau\}}\left(u^{m} \Delta \eta+u \frac{\partial \eta}{\partial t}\right) d x d t .
$$

We first observe that if $\eta_{1}, \eta_{2} \in \mathscr{A}$ and $\eta_{1}(x, \tau)=\eta_{2}(x, \tau)$ then

$$
\Lambda_{\tau}\left(\eta_{1}\right)=\Lambda_{\tau}\left(\eta_{2}\right) \text {. }
$$

To see this let $\eta(x, t)=\eta_{1}(x, t)-\eta_{2}(x, t)$ for $t>\tau$ and 0 otherwise. Pick $\psi \in C_{0}^{\infty}(\mathbf{R})$ with support in $[0, \infty)$ and $\int \psi d t=1$. Set for $\varepsilon>0$

$$
\eta_{\varepsilon}(x, t)=\int \eta(x, t-\varepsilon s) \psi(s) d s .
$$

Since $u$ is a weak solution $\Lambda_{\tau}\left(\eta_{\varepsilon}\right)=0$ for all $\varepsilon>0$. Notice next that $\partial \eta / \partial t \in$ $L^{\infty}$ and $\partial \eta_{\varepsilon} / \partial t \rightarrow \partial \eta / \partial t$ almost everywhere so that

$$
\Lambda_{\tau}(\eta)=\lim _{\varepsilon \rightarrow 0} \Lambda_{\tau}\left(\eta_{\varepsilon}\right)=0
$$

which easily yields (8).

We now set for $\gamma \in C_{0}^{\infty}(D)$

$$
\lambda_{\tau}(\gamma)=\Lambda_{\tau}(\eta)
$$

where $\eta$ is any function in $\mathscr{A}$ for which $\eta(x, \tau)=\gamma(x)$. The functional $\lambda_{\tau}$ is well defined by (8). We now claim that

$$
\lambda_{\tau}(\gamma) \leq 0
$$

for all nonnegative $\gamma \in C_{0}^{\infty}(D)$. To see this pick this time $\psi \in C_{0}^{\infty}(\mathbf{R})$ such that $\psi(0)=1$ and

$$
\psi^{\prime}(s) \geq 0 \text { for } s \leq 0 .
$$

Set next for $\varepsilon>0 \quad \psi_{\varepsilon}(s)=\psi(s / \varepsilon)$ and $\eta_{\varepsilon}(x, t)=\psi_{\varepsilon}(t-\tau) \gamma(x)$. Since $u$ is a weak solution we have

$$
\lambda_{\tau}(\gamma)=-\iint_{\Omega \cap\{t \leq \tau\}}\left(u^{m} \Delta \gamma(x) \psi_{\varepsilon}(t-\tau)+u \gamma(x) \frac{\partial \psi_{\varepsilon}(t-\tau)}{\partial t}\right) d x d t .
$$

From (10) follows

$$
\lambda_{\tau}(\gamma) \leq-\iint_{\Omega \cap\{t \leq \tau\}} u^{m} \Delta \gamma(x) \psi_{\varepsilon}(t-\tau) d x d t \rightarrow 0
$$

as $\varepsilon \rightarrow 0$, which yields (9). From this (7) immediately follows. To conclude the proof of the lemma we note that (6) follows by considering the trace with respect to slightly larger domains $D^{\prime}$ such that $D$ is compactly contained in $D^{\prime}$. We will next study the existence of a trace on the lateral sides of a cylinder in $\Omega$.

Lemma 3.2. Let $I=(a, b)$ be an interval and let $D \subset \mathbf{R}^{n}$ be a bounded domain with smooth boundary. Set $\omega=D \times(a, b), K=\bar{D}$ and assume that $K \times[a, b]$ is compact and contained in $\Omega$. Then there is a unique bounded and nonnegative measure $\mu$ on $S=\partial D \times(a, b)$ such that

$$
\iint_{\omega}\left(u^{m} \Delta \eta+u \frac{\partial \eta}{\partial t}\right) d x d t+\iint_{S} \frac{\partial \eta}{\partial n} d \mu=0
$$


for each $\eta \in \mathscr{B}$. Here $\partial / \partial n$ denotes differentiation with respect to the inward unit normal to $\partial D$ and $\mathscr{B}$ denotes the class of all

$$
\eta \in C_{0}^{\infty}(\Omega \cap\{(x, t): a<t<b\})
$$

with the property that $\eta(x, t)=0$ whenever $x \in \partial D$.

Proof. We begin by introducing the functional $\Lambda(\eta)$ for $\eta \in \mathscr{B}$ by setting

$$
\Lambda(\eta)=\iint_{\omega}\left(u^{m} \Delta \eta+u \frac{\partial \eta}{\partial t}\right) d x d t
$$

where $\omega=D \times(a, b)$. Choose now a $\psi \in C_{0}^{\infty}\left(\mathbf{R}^{n}\right)$ such that $\psi \geq 0$ and $\int \psi d x=1$. For $\eta \in \mathscr{B}$ and $\varepsilon>0$ set

$$
\eta_{\varepsilon}(x, t)=\varepsilon^{-n} \int_{D} \eta(\xi, t) \psi\left(\frac{x-\xi}{\varepsilon}\right) d \xi .
$$

We first observe that if $\eta \in \mathscr{B}$ with $\partial \eta / \partial n=0$ then since $u$ is a weak solution

$$
\Lambda(\eta)=\lim _{\varepsilon \rightarrow 0} \iint_{\omega}\left(u^{m} \Delta \eta_{\varepsilon}+u \frac{\partial \eta_{\varepsilon}}{\partial t}\right) d x d t=0
$$

If $\gamma \in C_{0}^{\infty}(S)$ we define $\lambda(\gamma)=\Lambda(\eta)$ by picking an $\eta \in \mathscr{B}$ such that $\partial \eta / \partial n=$ $\gamma$. By the above observation we have that $\lambda$ is well defined. Arguing as in the proof of Lemma 3.1 we see that it is enough to show $\lambda(\gamma) \leq 0$ whenever $\gamma \geq 0$. To see this set, for $\eta \in \mathscr{B}$,

$$
\tilde{\eta}= \begin{cases}\eta(x, t) & \text { for }(x, t) \in \omega, \\ 0 & \text { otherwise }\end{cases}
$$

Let $\kappa$ denote the characteristic function of the set $\omega$. If $\gamma=\partial \eta / \partial n \geq 0$ then we have the inequality $\Delta \tilde{\eta} \geq \kappa \Delta \eta$ in the sense of distributions. Define as before

$$
\tilde{\eta}_{\varepsilon}(x, t)=\varepsilon^{-n} \int_{D} \tilde{\eta}(\xi, t) \psi\left(\frac{x-\xi}{\varepsilon}\right) d \xi
$$

Since $u$ is a solution we have

$$
\lambda(\gamma) \leq \lim _{\varepsilon \rightarrow 0} \iint\left(u^{m} \Delta \tilde{\eta}_{\varepsilon}+u \frac{\partial \tilde{\eta}_{\varepsilon}}{\partial t}\right) d x d t=0
$$

which yields the lemma.

Next we want to study the regularity of the trace $\nu_{\tau}$.

Lemma 3.3. Let $I=(a, b)$ be an interval and let $D \subset \mathbf{R}^{n}$ be a bounded domain with smooth boundary. Set $\omega=D \times(a, b)$ and assume that $\bar{\omega}$ is contained in $\Omega$. For $a<\tau<b$ let $\nu_{\tau}$ be the trace defined by (7). Suppose $\psi$ is $a$ continuous function with support in $\omega$. Then

$$
\int_{D} \psi(x, t) d \nu_{t}
$$

is a continuous function of $t$ and

$$
\int\left(\int_{D} \psi(x, \tau) d \nu_{\tau}\right) d \tau=\iint u \psi d x d \tau
$$

Proof. From an approximation argument follows that it is enough to verify the lemma in the case when $\psi \in C_{0}^{\infty}$. In this case the continuity (13) is an 
immediate consequence of the definition (7). To see the identity (14) we set $q(x, t)=(t-a) \psi(x, t)$ and by a change in the order of integration we get that

$$
\begin{aligned}
& \int\left(\int_{D} \psi(x, \tau) d \nu_{\tau}\right) d \tau \\
& \quad=-\int\left(\iint_{D \cap\{t>\tau\}}\left(u^{m} \Delta \psi+u \frac{\partial \psi}{\partial t}\right) d x d t\right) d \tau \\
& \quad=\iint u \psi d x d \tau-\iint\left(u^{m} \Delta q+u \frac{\partial q}{\partial t}\right) d x d t=\iint u \psi d x d \tau,
\end{aligned}
$$

which completes the proof of the lemma.

We can now give our most general trace result.

Lemma 3.4. Let $I=(a, b)$ be an interval and let $D \subset \mathbf{R}^{n}$ be a bounded domain with smooth boundary. Set $K=\bar{D}$ and assume that $K \times[a, b]$ is compact and contained in $\Omega$. For $a<\tau<b$ let $\nu_{\tau}$ be the trace defined by (7) and set $\omega=D \times(a, b)$. Suppose $\psi \in C_{0}^{\infty}(\Omega)$ equals zero on $\partial D \times[a, b]$. Then

$$
\begin{aligned}
\int_{D} \psi(x, b) d \nu_{b}= & \int_{D} \psi(x, a) d \nu_{a}+\iint_{\omega}\left(u^{m} \Delta \psi+u \frac{\partial \psi}{\partial t}\right) d x d t \\
& +\iint_{\partial D \times(a, b)} \frac{\partial \psi}{\partial n} d \mu,
\end{aligned}
$$

where $\mu$ is defined in Lemma 3.2.

Proof. Pick $\eta_{k} \in C_{0}^{\infty}(\mathbf{R})$ with $0 \leq \eta_{k} \leq 1$ and support in $(a, b)$ such that $\eta_{k}^{\prime} d t \rightarrow d \delta_{a}-d \delta_{b}$ weakly as $k \rightarrow \infty$, where $\delta_{a}$ is the Dirac measure at $a$. We may also assume that $\lim _{k \rightarrow \infty} \eta_{k}(t)=1$ when $a<t<b$ and $\lim _{k \rightarrow \infty} \eta_{k}(t)=0$ when $t \in \mathbf{R} \backslash[a, b]$. Using $q=\eta_{k} \psi$ as a test function it follows from (11) that

$$
\iint_{\omega}\left(u^{m} \eta_{k} \Delta \psi+u \eta_{k} \frac{\partial \psi}{\partial t}\right) d x d t+\iint_{\partial D \times(a, b)} \eta_{k} \frac{\partial \psi}{\partial n} d \mu=-\iint_{\omega} u \eta_{k}^{\prime} \psi d x d t .
$$

Letting $k \rightarrow \infty$ and using the continuity result from Lemma (3.3) completes the proof of (15).

\section{Potential theoretical methods}

In this section we will continue to assume that $u \geq 0$ and $u \in L_{\text {loc }}^{m}(\Omega)$ where $\Omega$ is a domain in $\mathbf{R}^{n+1}$. Let $I=(a, b)$ be an interval and let $D \subset \mathbf{R}^{n}$ be a bounded domain with smooth boundary. Set $\omega=D \times(a, b)$ and assume that $\bar{\omega}$ is contained in $\Omega$.

We will also assume that $u$ solves the equation $\partial u / \partial t=\Delta u^{m}$ in the distribution sense. Now let $G$ be the Green function of $D$ and define $w$ in $\omega$ by $w(x, t)=\int_{D} G(x, y) d \nu_{t}$ where $\nu_{t}$ is the trace defined by (7).

As a first step in proving the continuity of $u$ we will establish that $w$ is locally bounded. This will be achieved by first noting that $w$ satisfies a parabolic equation (16) and then using a variant of the Moser [7] iteration technique introduced in Dahlberg and Kenig [4]. We begin by establishing a useful consequence of the Sobolev inequality. 
Lemma 4.1. Let $F \geq 0$ be smooth in $\omega$. Suppose $m>1$ is given and $K$ is compact with $K \subset D$. Then there is $a k>1$ and $a \theta>0$ such that if $H \geq 0$ and $\Delta F \geq-H$ then

$$
\begin{aligned}
\iint_{K \times[a, b]} F^{k m} d x d t \leq C\{ & \iint_{\omega} H^{m} d x d t \sup _{t \in[a, b]}\left(\int_{D} F(x, t) d x\right)^{m(k-1)} \\
& \left.+\operatorname{dist}(K, \partial D)^{-\theta} \sup _{t \in[a, b]}\left(\int_{D} F(x, t) d x\right)^{m k}\right\},
\end{aligned}
$$

where $C$ only depends on $\omega$.

Proof. Define $p$ by

$$
p= \begin{cases}\frac{n}{n-2 m} & \text { if } n>2 m, \\ 2 & \text { otherwise }\end{cases}
$$

and set $q=\frac{p}{p-1}$. For $k>1$ and $t \in[a, b]$ we have by Hölder's inequality that

$$
\int_{K} F^{k m}(x, t) d x \leq\left(\int_{K} F^{p m}(x, t) d x\right)^{1 / p}\left(\int_{K} F^{(k-1) q m}(x, t) d x\right)^{1 / q} .
$$

We now choose $k$ so that $(k-1) q m=1$. Define $f$ by $f=\max (F-G H, 0)$, where $G H(x, t)=\int_{D} G(x, y) H(y, t) d y$. Then $f$ is a nonnegative subharmonic function in $x$ for every $t \in[a, b]$ and $F \leq f+G H$. We have by Sobolev's inequality

$$
\left(\int_{K}(G H)^{p m}(x, t) d x\right)^{1 / p} \leq C \int_{D} H^{m}(x, t) d x
$$

Since $f$ is subharmonic in $x$ we have that

$$
f(\xi) \leq C \operatorname{dist}(K, \partial D)^{-n} \int_{D} f d x
$$

for all $\xi$ in $K$. Since $f \leq F$ we therefore have that

$$
\left(\int_{K} f^{p m}(x, t) d x\right)^{1 / p} \leq C \operatorname{dist}(K, \partial D)^{-n m}\left(\int_{D} F(x, t) d x\right)^{m} .
$$

Adding these estimates and integrating in $t$ completes the proof of the lemma.

For $\eta \in C_{0}^{\infty}(\omega)$ we now have by Lemma 3.2 and Lemma 3.3

$$
\iint_{\omega} \frac{\partial \eta}{\partial t} w d x d t=\iint_{\omega} \frac{\partial G \eta}{\partial t} u d x d t=\iint_{\omega} \eta u^{m} d x d t-\iint_{\partial D \times[a, b]} \frac{\partial G \eta}{\partial n} d \mu .
$$

We can rewrite this as

$$
\iint_{\omega} \frac{\partial w}{\partial t} \eta d x d t+\iint_{\omega} \eta u^{m} d x d t=\iint_{\partial D \times[a, b]} \frac{\partial G \eta}{\partial n} d \mu .
$$

Define now for $(x, t) \in \omega$ the function $h$ by

$$
h(x, t)=\iint_{\partial D \times[a, t]} \frac{\partial G(\xi, x)}{\partial n_{\xi}} d \mu .
$$


Then $\Delta h(x, t)=0$ and $\int_{D} h(x, t) \leq C$ for all $t \in(a, b)$ and if we set $q=w-h$ then $q$ satisfies the following relations in the distribution sense

$$
\frac{\partial q}{\partial t}+u^{m}=0, \quad \Delta q=-u
$$

It follows from the Harnack inequality for harmonic functions and the uniform integrability of $h$ that for every compact set $K \subset D$ that $\sup _{K \times[a, b]} h<\infty$. Fix $x_{0} \in D$ and set $B_{\rho}=\left\{x \in \mathbf{R}^{n}:\left|x-x_{0}\right|<\rho\right\}$. Assume that $\bar{B}_{\rho} \subset D$, $a<T<\tau<b$, and set

$$
S=B_{\rho} \times(\tau, b], \quad R=B_{\rho} \times(T, b] .
$$

Fix $M>0$ so large such that

$$
Q=q+M \geq 0 \text { in } B_{\rho} \times[a, b] .
$$

Lemma 4.2. Suppose $P$ is a smooth and convex function with a bounded derivative $p$ on $[0, \infty)$. Also suppose that $P(0)=p(0)=0$. Define $Z$ by $Z(t)=$ $\int_{0}^{t}(p(s))^{1 / m} d s$. Then

$$
\begin{aligned}
& \sup _{s \in[\tau, b]} \int_{B_{\rho}} P(Q(x, s)) d x+\iint_{S}(\max (-\Delta Z(Q), 0))^{m} d x d t \\
& \quad \leq C(\tau-T)^{-1} \iint_{R} P(Q) d x d t,
\end{aligned}
$$

where the constant $C$ can be taken to depend only on the dimension $n$.

Proof. We first remark that from the convexity of $P$ and the vanishing of $P$ and $p$ at 0 follows that both $P$ and $p$ are nonnegative and nondecreasing.

Let $0<r<\rho$ and let $\eta \in C^{\infty}\left(\mathbf{R}^{n+1}\right), 0 \leq \eta \leq 1$, be such that $\eta(x, t)=1$ if $(x, t) \in B_{r} \times[\tau, \infty)$ and $\eta(x, t)=0$ whenever $x \in \mathbf{R}^{n} \backslash B_{\rho}$ or $t \leq T$. We may also choose $\eta$ so that

$$
\left|\frac{\partial \eta}{\partial t}\right| \leq C(\tau-T)^{-1}
$$

where the constant $C$ can be taken to depend only on the dimension $n$.

For $s \in[\tau, b]$ set $S^{\prime}=B_{r} \times(\tau, s], R^{\prime}=B_{\rho} \times(T, s]$. Since $\partial Q / \partial t+u^{m}=0$ we have by multiplying with $p(Q) \eta^{2}$ and integrating over $R^{\prime}$

$$
\iint_{R^{\prime}} p(Q) \eta^{2} \frac{\partial Q}{\partial t} d x d t+\iint_{R^{\prime}} p(Q) \eta^{2} u^{m} d x d t=0 .
$$

An integration by parts therefore gives that

$$
\int_{B_{\rho}} P(Q(x, s)) \eta^{2} d x+\iint_{R^{\prime}} p(Q) \eta^{2} u^{m} d x d t=2 \iint_{R^{\prime}} P(Q) \eta \frac{\partial \eta}{\partial t} d x d t
$$

and hence it follows that

$$
\int_{B_{\rho}} P(Q(x, s)) \eta^{2} d x+\iint_{R^{\prime}} p(Q) \eta^{2} u^{m} d x d t \leq C(\tau-T)^{-1} \iint_{R} P(Q) d x d t .
$$

Since $s \in[\tau, b]$ and $r \in(0, \rho)$ were arbitrary we conclude that

$$
\sup _{s \in[\tau, b]} \int_{B_{\rho}} P(Q(x, s)) d x+\iint_{S} p(Q) u^{m} d x d t \leq C(\tau-T)^{-1} \iint_{R} P(Q) d x d t
$$


Observing that $\Delta Z(Q) \geq-p(Q)^{1 / m} u$ yields the proof of the lemma.

We have the following immediate consequence of Lemma (4.2).

Lemma 4.3. Let $\alpha \geq 0$ and set $\beta=\frac{\alpha+m}{m}$. Then we have the following estimate

$$
\begin{gathered}
(\alpha+1)^{m-1} \sup _{\tau<s<b} \int_{B_{\rho}} Q^{\alpha+1}(x, s) d x+\iint_{S}\left(\max \left(-\Delta Q^{\beta}, 0\right)\right)^{m} d x d t \\
\leq C(\alpha+1)^{m-1}(\tau-T)^{-1} \iint_{R} Q^{\alpha+1} d x d t,
\end{gathered}
$$

where $C$ can be taken to depend only on $m$ and $n$.

Proof. For $L>0$ let $p$ be defined by

$$
p(s)= \begin{cases}(\alpha+1) s^{\alpha}, & 0 \leq s \leq L, \\ (\alpha+1) L^{\alpha}, & \text { otherwise }\end{cases}
$$

Using Lemma 4.2 with $P(s)=\int_{0}^{s} p(t) d t$ and then letting $L \rightarrow \infty$ yields the lemma.

We can now prove the boundedness of $w$.

Lemma 4.4. Let $\Omega \subset \mathbf{R}^{n+1}$ be a domain and assume $u \geq 0$ belongs to $L_{\text {loc }}^{m}(\Omega)$, where $1<m<\infty$. Suppose $D \subset \mathbf{R}^{n}$ is a domain with $\bar{D} \times[a, b] \subset \Omega$. Suppose furthermore that $a<T<b$ and $B_{3 \rho}=\left\{x \in \mathbf{R}^{n}:\left|x-x_{0}\right|<3 \rho\right\} \subset D$. If $\partial u / \partial t=\Delta u^{m}$ in $\Omega$ then $w=G u$ is bounded in $R=B_{\rho} \times[T, b]$. Here $G$ is the Green function of $D$.

Proof. In what follows we will let $C$ denote various constants that only depend on $m, \rho, T, a$ or $b$.

Let $Q$ be defined by (19) and assume that $M$ is chosen so large that $Q \geq 1$ in $B_{3 \rho} \times[a, b]$. For $j=0,1, \ldots$ define $\alpha_{0}=0$ and $\alpha_{j+1}=k\left(\alpha_{j}+m\right)-1$, where $k$ is as in Lemma 4.1. Set $\beta_{j}=\left(\alpha_{j}+m\right) / m, \rho_{j}=\rho \frac{j+2}{j+1}, T_{j}=\frac{a+j T}{1+j}$, $B_{j}=B_{\rho_{j}}, I_{j}=\left(T_{j}, b\right], R_{j}=B_{j} \times I_{j}$ and

$$
A_{j}=\left(\iint_{R_{j}} Q^{\alpha_{j}+1} d x d t\right)^{1 /\left(\alpha_{j}+1\right)} .
$$

Since $u \in L_{\text {loc }}^{m}(\Omega)$ we have that $A_{0}<\infty$. Setting

$$
E_{j}=\sup _{s \in I_{j}} \int_{B_{j}} Q^{\alpha_{j}+1} d x
$$

we have from Lemma 4.1 and Lemma 4.3 that

$$
\begin{aligned}
& A_{j+1}^{\alpha_{j+1}+1}=\iint_{R_{j+1}} Q^{\alpha_{j+1}+1} d x d t=\iint_{R_{j+1}} Q^{k \beta_{j} m} d x d t \\
& \quad \leq C\left\{\left(\alpha_{j}+1\right)^{m-1} A_{j}^{\alpha_{j}+1} E_{j}^{\left(\alpha_{j}+m\right)(k-1) /\left(\alpha_{j}+1\right)}(j+1)^{2}+(j+1)^{\theta} E_{j}^{\left(\alpha_{j}+m\right) k /\left(\alpha_{j}+1\right)}\right\} .
\end{aligned}
$$

We next observe that $(j+1)^{\theta} \leq C\left(\alpha_{j}+1\right)^{m-1}$ so using Lemma (4.3) once more gives

$$
A_{j+1}^{\alpha_{j+1}+1} \leq C\left(\alpha_{j}+1\right)^{m-1}\left(A_{j}^{k\left(\alpha_{j}+m\right)-m+1}+A_{j}^{k\left(\alpha_{j}+m\right)}\right)(j+1)^{2} .
$$

Since $Q \geq 1$ in $B_{3 \rho} \times[a, b]$ we have

$$
A_{j+1} \leq\left(C\left(\alpha_{j}+1\right)^{m-1}\right)^{1 /\left(\alpha_{j+1}+1\right)} A_{j}(j+1)^{2 /\left(\alpha_{j+1}+1\right)}
$$


which easily gives that $\|Q\|_{L_{R}^{\infty}} \leq C A_{0}$ and the lemma is proved.

Lemma 4.5. Let $I=(a, b)$ be an interval and let $D \subset \mathbf{R}^{n}$ be a bounded domain with smooth boundary. Set $\omega=D \times I$ assume that $\bar{\omega}$ is compact and contained in $\Omega$. Then $w(x, t)$ and $\int_{a}^{t} u^{m}(x, s) d s$ are bounded in $\omega$. Furthermore, if $(x, t) \in \omega$ then

$$
w(x, t)=G \nu_{a}(x)-\int_{a}^{t} u^{m}(x, s) d s+h(x, t) .
$$

Here $h$ is bounded in $\omega$ and satisfies $\Delta h=0$.

Proof. We first observe that the relation (21) follows from (18), with $h$ given by

$$
h(x, t)=\iint_{\partial D \times(a, t)} \frac{\partial G(x, y)}{\partial n_{y}} d \mu(y, s) .
$$

Since

$$
\sup _{t \in I} \int_{D} h(x, t) d x<\infty
$$

it follows from Harnack's inequality that for every compact $K \subset D$

$$
h(x, t) \leq C<\infty, \quad(x, t) \in K \times I .
$$

From Lemma (4.4) it follows now that

$$
\sup _{(x, t) \in K \times I} \int_{a}^{t} u^{m}(x, s) d s<\infty .
$$

Let $\mathscr{M} \subset \mathbf{R}^{n}$ be a bounded domain such that $\bar{D} \subset \mathscr{M}$ and $\overline{\mathscr{M}} \times[a, b] \subset \Omega$. If $G^{\prime}$ denotes the Green function of $\mathscr{M}$ then $w \leq G^{\prime} u$ in $\omega$ which shows that $w$ is bounded in $\omega$. Since $\bar{D} \subset \mathscr{M}$ it follows from the above argument that

$$
\sup _{(x, t) \in D \times I} \int_{a}^{t} u^{m}(x, s) d s<\infty
$$

which yields the lemma.

We want next to study the regularity of $w$ in Sobolev norms. We recall that the Sobolev space $H_{0}^{1}(D)$ is the completion of $C_{0}^{\infty}(D)$ in the norm

$$
\|\eta\|=\left(\int_{D}|\nabla \eta|^{2} d x\right)^{1 / 2}
$$

We first observe that if $D \subset \mathbf{R}^{n}$ is a domain with $\bar{D} \times[a, b] \subset \Omega$ then $w=$ $G u=G \nu_{t}$ satisfies $w(\cdot, t) \in H_{0}^{1}(D)$ for all $t \in[a, b]$. This holds by the boundedness of $w$ since

$$
\int_{D}|\nabla w(x, t)|^{2} d x=\int_{D} w d \nu_{t}<\infty
$$

where $\nu_{t}$ is the trace defined by (7). For a function $f \in H_{0}^{1}(D)$ we set

$$
\mathscr{E}(f)=\int_{D}|\nabla f|^{2} d x
$$

We now want to show that $u \in L_{\mathrm{loc}}^{m+1}(\Omega)$. 
Theorem 4.6. Let $\Omega \subset \mathbf{R}^{n+1}$ be a domain. Let $u \in L_{\mathrm{loc}}^{m}(\Omega), m>1$. Suppose $u$ is a weak solution of the porous medium equation $\frac{\partial u}{\partial t}=\Delta u^{m}$ in $\Omega$. Then $u \in L_{\mathrm{loc}}^{m+1}(\Omega)$.

In order to show this, we need a preliminary result.

Lemma 4.7. Let $D \subset \mathbf{R}^{n}$ be a bounded domain with a smooth boundary and set $\omega=D \times(a, b)$ where $a<b$. Suppose that $w$ is smooth in a neighborhood of $\bar{\omega}$, satisfies $\partial w / \partial t \leq 0$ and $\Delta w \leq 0$. Assume also that $\int_{D}|\Delta w(x, t)| d x \leq M$ for all $t \in(a, b)$ and $|w| \leq M$. Then, for every compact set $K \subset D$ there is a constant $C=C(K, \omega)$ such that

$$
\iint_{S} \frac{\partial w}{\partial t} \Delta w d x d t \leq C M^{2}
$$

where $S=K \times(a, b)$.

Proof. We can assume, without loss of generality, that $w \geq 0$ in $\bar{\omega}$, and that $M=1$. Let us first assume that, in addition, $\left.w\right|_{\partial D \times[a, b]} \equiv 0$. Then

$$
\frac{\partial}{\partial t} \int_{D} w \Delta w=\int_{D} \frac{\partial w}{\partial t} \Delta w+w \frac{\partial}{\partial t} \Delta w=2 \int_{D} \frac{\partial w}{\partial t} \Delta w .
$$

Therefore,

$$
\int_{D} w(x, b) \Delta w(x, b)-\int_{D} w(x, a) \Delta w(x, a)=2 \iint_{D \times(a, b)} \frac{\partial w}{\partial t} \Delta w
$$

and hence the desired estimate follows in this case. Before passing to the general case, we note that there exists $p=p(n)>1$ such that, if $\widetilde{K} \subset \subset D$, we have

$$
\left(\int_{\widetilde{K}}|\nabla w(x, t)|^{p} d x\right) \leq C, \quad \text { for all } t \in(a, b),
$$

where $C=C(\widetilde{K})$. (This is standard, since $0 \leq w \leq 1, \int_{D}|\Delta w(x, t)| d x \leq 1$, and therefore we can take any $1<p<\frac{n}{n-1}$.)

Fix now $K \subset \subset D$, and pick $K \subset \subset \widetilde{D} \subset \subset D$, where $\widetilde{D}$ is a smooth domain, and for $\tau \in[0,1]$, a smooth one-parameter family of smooth domains $\widetilde{D}_{\tau}$, such that

(i) $K \subset \subset \widetilde{D}_{\tau}$ for all $\tau \in[0,1]$,

(ii) $\widetilde{D}_{\tau} \subset \subset \widetilde{D}_{\tau}^{\prime}$ if $\tau<\tau^{\prime}$,

(iii) $\widetilde{D}_{1}=\widetilde{D}$,

(iv) $U=\bigcup_{0 \leq \tau \leq 1} \widetilde{D}_{\tau}$ is an open neighborhood of $K$ in $D$,

and there exist diffeomorphisms $\theta_{\tau}: S^{n-1} \rightarrow \partial \widetilde{D}_{\tau}$

such that $(\tau, Q) \rightarrow \theta_{\tau}(Q)$ is a diffeomorphism of $(0,1) \times S^{n-1}$ onto $U$.

Now, for each $0 \leq \tau \leq 1$, we define

$$
w_{\tau}=\left\{\begin{array}{l}
w \text { in } \widetilde{D}_{\tau}, \text { harmonic in } \widetilde{D} \backslash \widetilde{D}_{\tau}, \text { with boundary } \\
\text { values } w \text { on } \partial \widetilde{D}_{\tau}, 0 \text { on } \partial \widetilde{D} .
\end{array}\right.
$$


Clearly, each $w_{\tau}$ is continuous, superharmonic and $0 \leq w_{\tau} \leq w \leq 1$. Moreover, because of our construction of $w_{\tau}, \partial w_{\tau} / \partial t \leq 0$. By (22), we have

$$
\begin{aligned}
\iint_{\widetilde{D} \times(a, b)} \frac{\partial w_{\tau}}{\partial t} \Delta w_{\tau} & \leq \frac{1}{2} \int_{D} w_{\tau}(x, a)\left|\Delta w_{\tau}(x, a)\right| d x \\
& \leq \frac{1}{2} \int_{\widetilde{D}}\left|\Delta w_{\tau}(x, a)\right| d x .
\end{aligned}
$$

Moreover,

$$
\iint_{K \times(a, b)} \frac{\partial w}{\partial t} \Delta w=\iint_{K \times(a, b)} \frac{\partial w_{\tau}}{\partial t} \Delta w_{\tau} \leq \iint_{\widetilde{D} \times(a, b)} \frac{\partial w_{\tau}}{\partial t} \Delta w_{\tau}
$$

The proof will be concluded if we show that

$$
\int_{1 / 2}^{3 / 4} \int_{\widetilde{D}}\left|\Delta w_{\tau}(x, a)\right| d x d \tau \leq C .
$$

In order to show this, note that

$$
\Delta w_{\tau}=(\Delta w) \chi_{\widetilde{D}_{\tau}}+\left(\frac{\partial w}{\partial n_{\tau}}-\frac{\partial w_{\tau}}{\partial n_{\tau}}\right) d \sigma_{\partial \widetilde{D}_{\tau}},
$$

where $\chi_{\widetilde{D}_{\tau}}$ is the characteristic function of $\widetilde{D}_{\tau}$, and $\partial / \partial n_{\tau}$ denotes differentiation along the normal to $\partial \widetilde{D}_{\tau}$. The first term is obviously under control. For the second one, note that

$$
\int_{\widetilde{D}}\left|\frac{\partial w}{\partial n_{\tau}} d \sigma_{\partial \widetilde{D}_{\tau}}\right| \leq \int_{\partial \widetilde{D}_{\tau}}\left|\frac{\partial w}{\partial n_{\tau}}\right| d \sigma \leq \int_{\partial \widetilde{D}_{\tau}}|\nabla w| d \sigma
$$

which, upon integration in $\tau$, is under control by (24)(iv) and (23), while

$$
\begin{aligned}
\int_{\widetilde{D}}\left|\frac{\partial w_{\tau}}{\partial n_{\tau}}\right| d \sigma_{\partial \widetilde{D}_{\tau}} & \leq C\left(\int_{\partial \widetilde{D}_{\tau}}\left|\frac{\partial}{\partial n_{\tau}} w_{\tau}\right|^{p} d \sigma\right)^{1 / p} \\
& \leq C\left(\int_{\partial \widetilde{D}_{\tau}}\left|\nabla_{T} w_{\tau}\right|^{p} d \sigma\right)^{1 / p},
\end{aligned}
$$

where $p$ is as in (23) and $\nabla_{T}$ denotes tangential differentiation. The last estimate follows by standard regularity estimates for the Dirichlet problem in the smooth domain $\widetilde{D} \backslash \widetilde{D}_{\tau}, \frac{1}{2}<\tau<\frac{3}{4}$. But $\nabla_{T} w_{\tau}=\nabla_{T} w$, and the proof is concluded as before.

We can now prove Theorem 4.6.

Proof of Theorem 4.6. Let $D \subset \mathbf{R}^{n}$ be a bounded domain with a smooth boundary and set $\omega=D \times(a, b)$ where $a<b$. Assume that $\bar{\omega} \subset \Omega$ and let $K$ be a compact set with $K \subset D$. Let $\nu$ denote the trace of $u$ on $\{(x, t) \in \Omega: t=a\}$ and set

$$
v(x, t)=G \nu(x)-\int_{a}^{t} u^{m}(x, s) d s .
$$

Pick $\varphi \in C_{0}^{\infty}\left(\mathbf{R}^{n+1}\right)$ such that $\varphi>0$ and $\iint \varphi d x d t=1$. Define the approximation of identity $\lambda_{\varepsilon}$ by

$$
\lambda_{\varepsilon} v(x, t)=\iint \varphi_{\varepsilon}(\xi, \tau) v(x-\xi, t-\tau) d \xi d \tau
$$


where $\varphi_{\varepsilon}(x, t)=\varepsilon^{-1-n} \varphi(x / \varepsilon, t / \varepsilon)$. Notice that $\frac{\partial}{\partial t} \lambda_{\varepsilon} v=-\lambda_{\varepsilon} u^{m} \leq 0$ and $\Delta \lambda_{\varepsilon} v=-\lambda_{\varepsilon} u \leq 0$. It now follows from Lemma 4.10 that

$$
\iint_{K \times(a, b)} \lambda_{\varepsilon} u \lambda_{\varepsilon} u^{m} d x d t \leq C
$$

where $C$ is independent of $\varepsilon$ as $\varepsilon \downarrow 0$. By letting $\varepsilon \downarrow 0$ it follows from Fatou's lemma that

$$
\iint_{K \times(a, b)} u^{m+1} d x d t<\infty
$$

Since $K$ was arbitrary the theorem follows.

\section{REGULARITY OF WEAK SOLUTIONS}

In order to prove the smoothness of a weak solution $u$ of the porous medium equation we begin by analyzing the effect of smoothing $u$ by an approximation of the identity $\left\{T_{\varepsilon}\right\}$. Here the operator $T_{\varepsilon}$ is defined by

$$
T_{\varepsilon} f(x, t)=\iint f(x-\xi, t-\tau) \eta_{\varepsilon}(\xi, \tau) d \xi d \tau
$$

where $\eta_{\varepsilon}(x, t)=\varepsilon^{-n-1} \eta(x / \varepsilon, t / \varepsilon)$ and $0 \leq \eta \in C_{0}^{\infty}\left(\mathbf{R}^{n+1}\right)$. We also assume that $\iint \eta d x d t=1$ and that the support of $\eta$ is contained in a sufficiently small neighborhood of the origin.

We assume now that $\bar{B} \times[a, b] \subset \Omega$ where $B=B\left(x_{0}, r\right)=\left\{x \in \mathbf{R}^{n}\right.$ : $\left.\left|x-x_{0}\right|<r\right\}$. We set $\omega=B \times(a, b)$. We recall that the trace $\nu, \mu$ satisfies by (15)

$$
\begin{aligned}
\int_{B} \psi(x, b) d \nu_{b}=\int_{B} \psi(x, a) d \nu_{a} \\
\quad+\iint_{\omega}\left(u^{m} \Delta \psi+u \frac{\partial \psi}{\partial t}\right) d x d t+\iint_{\partial B \times(a, b)} \frac{\partial \psi}{\partial n} d \mu
\end{aligned}
$$

for all $\psi \in C_{0}^{\infty}\left(\mathbf{R}^{n+1}\right)$ with $\psi=0$ on $\partial B \times \mathbf{R}$.

We will let $G$ denote the Green function of $B$ and we set as before $w=G u$.

Lemma 5.1. There is a continuous weak solution $u^{*} \geq 0$ of the porous medium equation $\partial u^{*} / \partial t=\Delta\left(u^{*}\right)^{m}$ in $\omega$ such that $u=u^{*}$ a.e in $\omega$.

Proof. Set $\delta(x)=\operatorname{dist}(x, \partial B)$. We begin by showing the existence of a continuous weak solution $u^{*} \geq 0$ in $\omega$ that satisfies

$$
\iint_{\omega}\left(u^{*}\right)^{m} d x d t<\infty
$$

and

$$
\sup _{t \in(a, b)} \int_{B} \delta(x) u^{*}(x, t) d x<\infty .
$$

Furthermore $u^{*}$ satisfies

$$
\begin{aligned}
& \int_{B} \psi(x, b) u^{*}(x, b) d x=\int_{B} \psi(x, a) d \nu_{a} \\
& \quad+\iint_{\omega}\left(\left(u^{*}\right)^{m} \Delta \psi+u^{*} \frac{\partial \psi}{\partial t}\right) d x d t+\iint_{\partial B \times(a, b)} \frac{\partial \psi}{\partial n} d \mu
\end{aligned}
$$

for all $\psi \in C_{0}^{\infty}\left(\mathbf{R}^{n+1}\right)$ with $\psi=0$ on $\partial B \times \mathbf{R}$. 
We will from now on limit $\varepsilon$ to the range $\varepsilon \in\left(0, \varepsilon_{0}\right)$ where $\varepsilon_{0}$ is chosen sufficiently small. We set $B_{\varepsilon}=B\left(x_{0}, r-\varepsilon\right), \omega_{\varepsilon}=B_{\varepsilon} \times(a, b)$ and define for $(x, t) \in \omega_{\varepsilon}, u_{\varepsilon}(x, t)=T_{\varepsilon} u(x, t)$. Let $G_{\varepsilon}$ denote the Green function of $B_{\varepsilon}$ and set $w_{\varepsilon}=G_{\varepsilon} u_{\varepsilon}$. We define $u_{\varepsilon}^{*}$ as the solution of the porous medium equation in $\omega_{\varepsilon}$ with data $u_{\varepsilon}^{*}=u_{\varepsilon}$ on $\bar{B}_{\varepsilon} \times\{a\}$ and $u_{\varepsilon}^{*}=\left(T_{\varepsilon} u^{m}\right)^{1 / m}$ on $\partial B_{\varepsilon} \times[a, b)$. Let $\delta_{\varepsilon}(x)=\operatorname{dist}\left(x, \partial B_{\varepsilon}\right)$. We now claim that there is a constant $C$ such that for all $\varepsilon \in\left(0, \varepsilon_{0}\right)$ we have that

$$
\sup _{t \in(a, b)} \int_{B_{\varepsilon}} \delta_{\varepsilon}(x) u_{\varepsilon}^{*}(x, t) d x \leq C
$$

and

$$
\iint_{\omega_{\varepsilon}}\left(u_{\varepsilon}^{*}\right)^{m} d x d t \leq C .
$$

It is easily seen that if $\psi \in C_{0}^{\infty}\left(\mathbf{R}^{n}\right), \psi=0$ on $\partial B$, and setting

$$
\psi_{\varepsilon}(x)=\psi\left(\frac{\left(x-x_{0}\right) r}{r-\varepsilon}+x_{0}\right)
$$

then

$$
\lim _{\varepsilon \downarrow 0} \int_{B_{\varepsilon}} \psi_{\varepsilon}(x) u_{\varepsilon}^{*}(x, a) d x=\int_{B} \psi(x) d \nu_{a}(x) .
$$

Also Lemma (3.2) shows that

$$
\lim _{\varepsilon \downarrow 0} \iint_{\partial B_{\varepsilon} \times(a, b)} \frac{\partial \psi}{\partial n}\left(u_{\varepsilon}^{*}\right)^{m} d \sigma d t=\iint_{\partial B \times(a, b)} \frac{\partial \psi}{\partial n} d \mu .
$$

If $\psi \in C_{0}^{\infty}\left(\mathbf{R}^{n+1}\right), \psi=0$ on $\partial B \times \mathbf{R}$ then for all sufficiently small $\varepsilon$

$$
\begin{aligned}
& -\iint_{\partial B_{\varepsilon} \times(a, b)} \frac{\partial \psi}{\partial n}\left(u_{\varepsilon}^{*}\right)^{m} d \sigma d t=\int_{B_{\varepsilon}} \psi(x, a) u_{\varepsilon}^{*}(x, b) d x \\
& \quad+\iint_{\omega_{\varepsilon}}\left(\left(u_{\varepsilon}^{*}\right)^{m} \Delta \psi+u_{\varepsilon}^{*} \frac{\partial \psi}{\partial t}\right) d x d t-\int_{B_{\varepsilon}} \psi(x, b) u_{\varepsilon}^{*}(x, b) d x .
\end{aligned}
$$

Using Proposition (2.1) we see that the estimates (28) and (29) follow from (30). It follows from (28) and the pointwise bounds in Dahlberg and Kenig [4] that $\left\{u_{\varepsilon}^{*}\right\}$ is uniformly bounded on compact subsets of $\omega$. Therefore by the regularity results of Sacks [8] it follows that $\left\{u_{\varepsilon}^{*}\right\}$ is equicontinuous on compact subsets of $\omega$. Hence there is a continuous weak solution $u^{*}$ in $\omega$ of the porous medium equation such that a subsequence of $u_{\varepsilon}^{*}$, which we also denote by $u_{\varepsilon}^{*}$ converges to $u^{*}$ as $\varepsilon \downarrow 0$ and $u^{*}$ satisfies (25) and (26). By taking the limit in (30) it also follows that (27) holds.

Define $h_{\varepsilon}$ as the solution of the equation

$$
\begin{cases}\Delta h_{\varepsilon}=0 & \text { in } \omega_{\varepsilon}, \\ h_{\varepsilon}=T_{\varepsilon} u^{m} & \text { on } \partial B_{\varepsilon} \times(a, b) .\end{cases}
$$

Using this notation we see that

$$
\frac{\partial w_{\varepsilon}}{\partial t}=G_{\varepsilon} T_{\varepsilon} \frac{\partial u}{\partial t}=G_{\varepsilon} \Delta T_{\varepsilon} u^{m}=h_{\varepsilon}-T_{\varepsilon} u^{m} .
$$

Setting $w_{\varepsilon}^{*}=G_{\varepsilon} u_{\varepsilon}^{*}$ we have that

$$
\frac{\partial w_{\varepsilon}^{*}}{\partial t}=G_{\varepsilon} \Delta\left(u_{\varepsilon}^{*}\right)^{m}=h_{\varepsilon}-\left(u_{\varepsilon}^{*}\right)^{m} .
$$


For $f$ a smooth function in $B_{\varepsilon}$ set $\mathscr{E}_{\varepsilon}(f)=\int_{B_{\varepsilon}}|\nabla f|^{2} d x$. Define $E_{\varepsilon}=w_{\varepsilon}^{*}-w_{\varepsilon}$ and set $e_{\varepsilon}(t)=\mathscr{E}_{\varepsilon}\left(E_{\varepsilon}(\cdot, t)\right)$. We will first show $\lim _{\varepsilon \downarrow 0} e_{\varepsilon}(t)=0$ for all $t \in$ $(a, b)$. We first observe that $T_{\varepsilon} u \rightarrow u$ in $L^{m+1}(\omega)$ by Theorem 4.6. Since $E_{\varepsilon}=0$ on $B_{\varepsilon} \times\{a\}$ we have

$$
\iint_{\omega_{\varepsilon}} \frac{\partial E_{\varepsilon}}{\partial t} \Delta E_{\varepsilon} d x d t=-\frac{1}{2} \int_{a}^{b} \frac{\partial e_{\varepsilon}}{\partial t} d t \leq 0 .
$$

Defining $L_{\varepsilon} \geq 0$ by $L_{\varepsilon}^{m+1}=\iint_{\omega_{\varepsilon}}\left(u_{\varepsilon}^{*}\right)^{m+1} d x d t$ we have that

$$
\begin{aligned}
L_{\varepsilon}^{m+1} & =\iint_{\omega_{\varepsilon}}\left(u_{\varepsilon}^{*}\right)^{m+1} d x d t \leq \iint_{\omega_{\varepsilon}}\left(u_{\varepsilon}^{*}\right)^{m} T_{\varepsilon} u+u_{\varepsilon}^{*} T_{\varepsilon} u^{m} \\
& \leq C\left(L_{\varepsilon}+L_{\varepsilon}^{m}\right) .
\end{aligned}
$$

Hence $L_{\varepsilon}$ is bounded as $\varepsilon \downarrow 0$ and we may assume that $u_{\varepsilon}^{*} \rightarrow u^{*}$ and $\left(u_{\varepsilon}^{*}\right)^{m} \rightarrow$ $\left(u^{*}\right)^{m}$ weakly in $L^{m+1}(\omega)$ and $L^{(m+1) / m}(\omega)$ respectively. We have now for every $T \in(a, b)$ that

$$
\begin{aligned}
0 & \leq e_{\varepsilon}(T)=2 \iint_{B_{\varepsilon} \times(a, T)}\left(u_{\varepsilon}-u_{\varepsilon}^{*}\right)\left(\left(u_{\varepsilon}^{*}\right)^{m}-T_{\varepsilon}\left(u^{m}\right)\right) d x d t \\
& \rightarrow 2 \iint_{B \times(a, T)}\left(u-u^{*}\right)\left(\left(u^{*}\right)^{m}-u^{m}\right) d x d t \leq 0 \quad \text { as } \varepsilon \downarrow 0 .
\end{aligned}
$$

Hence the lemma follows.

Theorem 1.1 is an immediate consequence of the lemma.

\section{REFERENCES}

1. D. Aronson and L. Caffarelli, The initial trace of a solution of the porous medium equation, Trans. Amer. Math. Soc. 280 (1983), 351-366.

2. Ph. Benilan, M. G. Crandall and M. Pierre, Solutions of the porous medium equation under optimal conditions on initial values, Indiana Univ. Math. J. 33 (1984), 51-87.

3. L. A. Caffarelli and A. Friedman, Regularity of the free boundary of a gas flow in an ndimensional porous medium, Indiana U. Math. J. 252 (1980), 361-391.

4. B. E. J. Dahlberg and C. Kenig, Non-negative solutions to the porous medium equation, Comm. Partial Differential Equations 9 (1984), 409-437.

5 . _ Nonnegative solutions of generalized porous medium equations, Rev. Mat. Iberoamericana 2 (1986), 267-305.

6. E. B. DiBenedetto and A. Friedman, Hölder estimates for nonlinear degenerate parabolic systems, J. Reine Angew. Math. 357 (1985), 1-22.

7. J. Moser, On Harnack's theorem for elliptic differential equations, Comm. Pure Appl. Math. 14 (1961), 577-591.

8. P. E. Sacks, Continuity of solutions of a singular parabolic equation, Nonlinear Analysis 7 (1983), 387-409.

9. E. M. Stein, Singular integrals and differentiability properties of functions, Princeton Univ. Press, 1970.

Department of Mathematics, Chalmers University of Technology, S 141296 GoteBORG, SWEDEN

Department of Mathematics, University of Chicago, Chicago, Illinois 60637 